\title{
PREPARÁNDOSE PARA LA MIRADA DEL OTRO: ORIGEN, SIGNIFICADOS Y FUNCIONES DE LOS MÁS ANTIGUOS COLGANTES HECHOS CON CONCHAS MARINAS
}

José Manuel Vázquez Varela

Universidade de Santiago de Compostela

RESUMEN

Se presenta un análisis de los más antiguos colgantes hechos con conchas marinas con el fin de conocer sus orígenes, significados y funciones en el momento de su aparición que se puede datar hace más de 125.000 años. Entre otros se puede señalar su carácter simbólico.

Palabras clave: colgantes, simbolismo, Paleolítico, funciones, significados.

\section{ABSTRACT}

An analysis of the earlier seashell personal ornaments is made in order to know the origins, meanings and functions at the time of its appearance around 125.000 years ago. Their symbolic nature is possible.

Keywords: personal ornament, symbolism, Paleolithic, functions, meanings.

\section{Introducción}

La mirada es un instrumento privilegiado de recogida de información del entorno y de trasmisión de actitudes y emociones del que mira, por lo que en las distintas culturas humanas hay variadas formas de prepararse ante la mirada del otro, tanto como para protegerse de sus intenciones como para enviar un mensaje determinado sobre las circunstancias propias que se desean resaltar.

Como lo que se percibe es el cuerpo del otro este se adecua a los deseos y necesidades de comunicación de la persona mediante la ocultación de aquello que no se quiere comunicar o bien la exhibición intencional de lo que se quiere trasmitir. Para ello, tanto para proteger de la mirada del otro como para enviar de un modo selectivo aquello que interesa que sea apreciado de un modo claro y prioritario, se acude a varias técnicas de modificación cultural del cuerpo propio, transitorias o permanentes tales como el vestido, el tatuaje, la escarificación, mutilaciones, modificaciones de la anatomía, o el empleo de objetos adecuados como colgantes, joyas, adornos, etc.

Aquí se presenta un estudio sobre uno de los medios culturales de comunicación, que tomando como soporte el cuerpo humano modifican el simple mensaje natural de sus características biológicas, tal cual es el caso de los colgantes que emplean como cuentas conchas de moluscos marinos y que por ahora son uno de los documentos más antiguos seguros sobre el empleo de medios culturales de transmisión de información ante la mirada del otro.

\section{El origen de los colgantes hechos con conchas}

Los datos más seguros sobre el empleo de conchas de moluscos marinos para la elaboración de cuentas de colgantes se documentan en una amplia zona de África del Norte y del Sur y del Próximo Oriente con una cronología de 


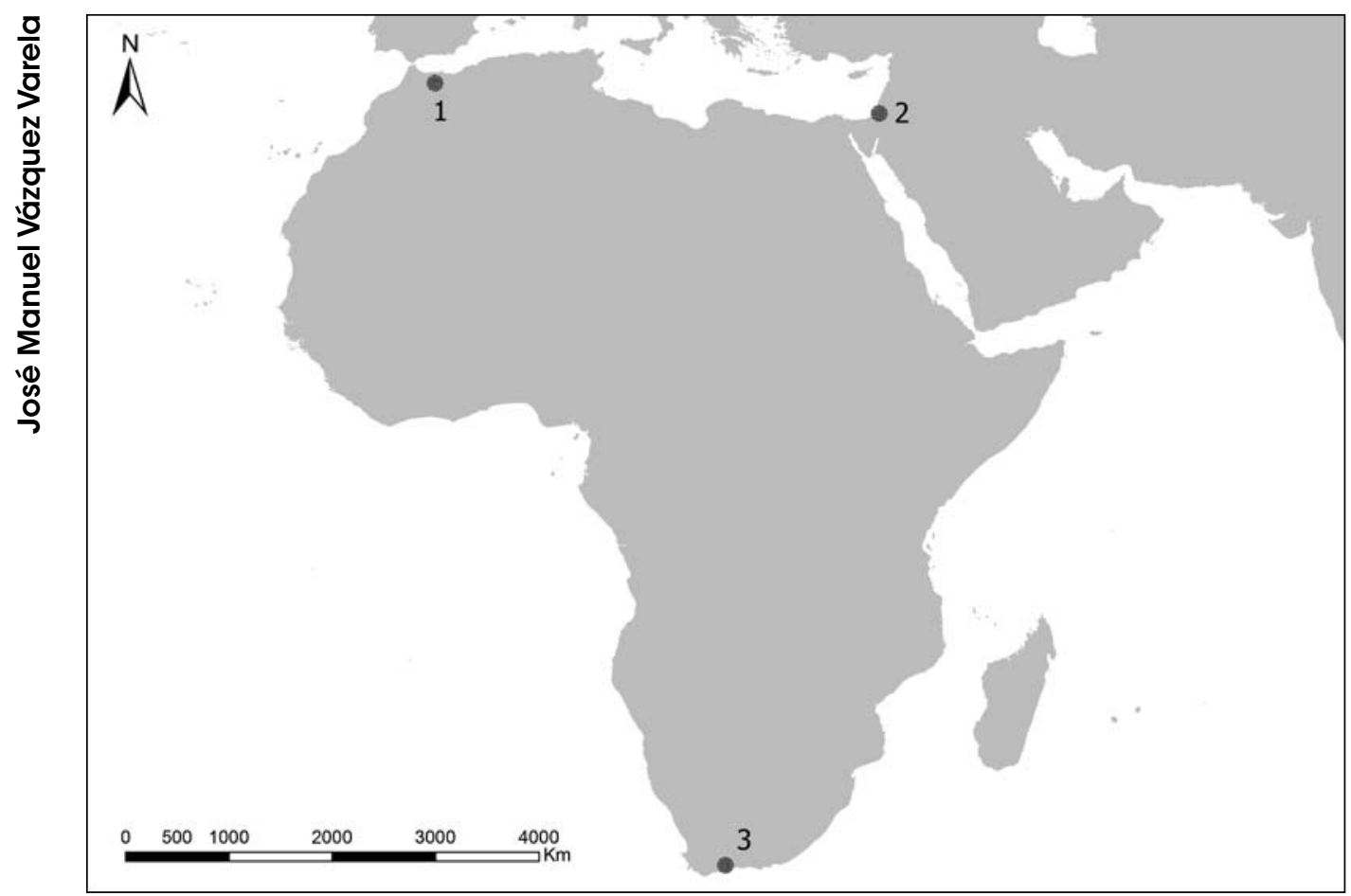

Fig. 1. Yacimientos con los collares de conchas más antiguos. 1. Marruecos y Argelia. 2. Próximo Oriente. 3. Sudáfrica

algo menos de 125.000 años $^{1}$. El hecho de que se encuentren tan dispersos en este momento permite pensar que su antigüedad es mayor en los que usan las conchas como materia básica y que posiblemente han existido con anterioridad otros hechos con materia orgánica perecedera, que por el momento no se ha podido documentar con seguridad, lo que podrá ser comprobado cuando se aumente el número de excavaciones en yacimientos más antiguos, ya que las realizadas hasta ahora son muy escasas y poco representativas de una etapa tan amplia como la comprendida por el Pleistoceno Medio y Superior.

Las conchas por su simetría y colores son fácilmente perceptibles por el cerebro donde producen una placentera sensación estética, por ello en muchas culturas tienen el valor de un bien de prestigio, que se adquiere a veces a través de largas cadenas de intercambio y su uso suele estar vinculado con variados significados y funciones entre las que se encuentran, entre otras el de ser un elemento indicador de la naturaleza social de su portador, protector contra el mal exterior, especialmente del mal de ojo, y también una exposición de diversas creencias y valores ${ }^{2}$.

\section{¿Qué son los colgantes?}

Los colgantes son objetos con varias posibles funciones y significados que suelen llevarse colgados de alguna parte del cuerpo o prendidos de los vestidos.

Una gran parte de ellos tiene uno o varios agujeros para facilitar su suspensión lo cual facilita su identificación, pero no por ello se ha de rechazar de esta categoría objetos de tamaño y peso reducido, que por su forma pudieran ser tales pues pueden ir envueltos en una piel, una tela de origen vegetal o animal o cualquier tipo de materia orgánica que pendería de un hilo o cordel. Así son varios colgantes de finalidad protectora consistentes en una pequeña bolsita como un escapulario que puede contener una estampa, un objeto religioso como un fragmento de piedra de ara de un altar, un texto evangé- 
lico, un escrito ritual, algunas plantas de poderes o simbolismos especiales, etc.

Por tanto el concepto de colgante es mucho más amplio que el que se limita a las piezas con agujero para pasar el hilo de suspensión.

Por otra parte a menudo no podemos precisar de qué tipo de colgante se trata pues puede ser desde un adorno para el pelo, pendiente, nariguera, collar, brazalete, pulsera, anillo, colgar de las rodillas, tobillera o ajorca, ir encajado por diversos procedimientos en diferentes partes del cuerpo, etc.

El contexto de los hallazgos y el estudio de sus huellas de uso ayudan a entender su naturaleza y funciones.

Una de las paradojas de la teoría de la comunicación es que a veces su ausencia intencional resulta comunicativa. Así por ejemplo, cuando una de dos personas, que se saludan habitualmente, no saluda a la otra a propósito este gesto consciente se puede entender como una ruptura intencionada de la comunicación.

En el caso de los colgantes el no llevarlos puede corresponder a una decisión deliberada, tal como ocurre en el caso de algunos grupos religiosos que renuncian al embellecimiento artificial mediante joyas como señal de austeridad.

También hay que tener en cuenta que los tatuajes, que pueden ser a veces auténticos y complejos códigos expresivos, raramente dejan huella y no tenemos restos de ellos para la época que nos ocupa.

Otra cuestión y no de menor interés, es que puede que parte de los colgantes fuesen llevados debajo de los vestidos de modo que no se percibiesen desde el exterior y así pierden su dimensión comunicativa, pero no por ello dejan de señalar un vínculo entre el devoto que lleva una medalla o escapulario y la figura religiosa a la que se encomienda, o un amuleto que le transmite su poder.

\section{¿De qué depende el poder del colgante?}

Las funciones y significados concretos de los colgantes están relacionados de un modo u otro con los siguientes atributos: forma, materia prima, su procedencia local o exótica, color, brillo, tacto, dureza, trasparencia, olor, maleabilidad, valor social y económico, que depende del prestigio otorgado al material por las propiedades anteriores, por su escasez o abundancia y el trabajo empleado en su elaboración entre otros factores.

\section{¿Qué funciones y significados tienen los colgantes?}

Son realmente muy variadas en las distintas culturas conocidas a través de los registros histórico y etnográfico y entre ellas se pueden señalar como más relevantes las siguientes:

Adecuan al cuerpo como escenario de la representación simbólica a través de los objetos que lleva como puede suceder con una prenda.

Desde el punto de vista etológico un collar en el que aparezcan a la vista dientes ofrece la perspectiva de una segunda boca lo cual por ser un aumento aparente del tamaño y de la cantidad de los órganos de su portador sirve como elemento disuasorio.

Los elementos que componen el collar indican el tipo de animales que ha capturado el que lo ostenta y por tanto de aquellos animales a los que ha sometido y dar cuenta así de su poder como cazador, lo que puede al tiempo ser indicativo de una fuerza o habilidad más amplia e intensa, que va más allá de lo común entre los humanos, como por ejemplo como que a mayores de su habilidad cinegética tiene poder, gracia, suerte, baraca, etc.

A veces indican su vinculación simbólica con los animales de los que se exhiben los dientes mediante la indicación de su filiación, de propiedades en común, de vínculos simbólicos que los unen, etc. Es posible que los dientes o partes de animales indiquen que el portador tiene a mayores de las vinculaciones simbólicas o metafóricas con ellos también sea depositario de sus poderes.

La presencia de restos de animales de diferentes tipos, herbívoros, carnívoros, terrestres, marinos, fluviales, aves, etc. es indicativa de algún tipo de relación real o simbólica con otras dimensiones de los mundos reales e imaginarios los que aquellos pertenecen. 
Dentro del contexto de la vida social a menudo señalan los diferentes círculos a los que pertenece el individuo, desde su identidad personal hasta la étnica pasando por la familiar.

En ocasiones indica el valor, la capacidad, el estatus, su grado de iniciación y de poder, la disponibilidad para ejercer determinadas funciones, en que etapa de tránsito de la vida se encuentra, su relación con el entorno ambiental y el simbólico con lo que entendemos como imaginario, recuerdo sentimental o funcional de una aventura.

También puede ser expresivo de sus sentimientos y de su psicología.

Desde la perspectiva de lo religioso a menudo reflejan una religión o creencia concreta o un valor mágico o religioso de su portador. Puede ser un objeto indicativo de poder protector, ofensivo, curativo, un modo de controlar un poder especial, la vinculación con este último, etc. Cuando va oculto debajo de la ropa puede ser un protector.

Oculta o disfraza a quién lo lleva visible, ya que en cierto modo lo sustituye al centralizar la mirada del otro en el colgante. Resalta el cuerpo y lo complementa con un añadido.

Como mero ornato, si es que existe esta categoría por sí misma separada de otras, puede tener dos funciones: a) Ponerse algo bello que provoca un placer estético cuando el que lo lleva puede verse asimismo con la pieza puesta o recibe alabanzas por ella, o por lo bien que le queda. b) Una forma de mostrar la belleza a otros a través de esta comunicación que puede tener múltiples funciones para establecer diferentes posibles vínculos, ser punto de atracción de la mirada del otro y empleado por tanto en el cortejo o en las relaciones sociales como un medio de comunicación simbólico.

A veces son indicativos de un sistema de numeración y objetos empleados en intercambios por lo que el llevarlos encima sería una invitación al trato, y un modo cómodo de exhibirlos, e incluso de indicar una de las actividades de su usuario.

Su papel en el conjunto de los elementos culturales que acompañan al cuerpo puede ser superfluo o esencial, combinar o no funcional o simbólicamente con el vestido, el cuerpo, el cubrecabezas, etc. y remplazar aumentar o disminuir el valor, las funciones y significados de otras manipulaciones del cuerpo como el vestido, el tatuaje, las escarificaciones, las mutilaciones, etc.

\section{Visión analítica de conjunto de los col- gantes}

A la luz de los paralelos etnológicos e históricos y por el valor que de acuerdo con ellos tiene cada uno de los componentes y por el de todos ellos en conjunto dispuestos como collares o como colgantes pueden tener un valor como protector contra los males de tipo simbólico. Si bien precisar su función y significados es un riesgo que nada más que nos debe conducir a formular una hipótesis podemos hacer un intento con ciertos visos de probabilidad: se puede tratar en algunos casos de objetos que tienen un valor protector contra los males que proceden del exterior de la persona o de uno en concreto de los males imaginarios, que es el más extendido en el tiempo y en el espacio a lo largo de culturas de muy distinta complejidad y organización: el mal de ojo. Se trata de una protección contra un mal que se trasmite desde el poseedor de ese poder negativo a través de la mirada que causa numerosos trastornos al que la recibe sin la protección adecuada. Los datos esenciales que apoyan esta hipótesis son los siguientes: la mirada es la forma de comunicación más directa y que trasmite mejor las intenciones, en etología la mirada directa es una forma de amenaza y desafío y la mayor parte de los elementos que aparecen en los colgantes tienen todavía un valor protector contra el mal de ojo en numerosas culturas de muy distinta complejidad y posición geográfica.

La función de los amuletos es la de desviar la mirada maligna de los ojos del que la recibe para sí evitar sus efectos. Mecanismo parecido tienen figuras de culturas conocidas a través del registro etnológico e histórico en las que determinados temas o gestos provocan horror con lo cual se aleja a las fuerzas malignas.

Por todo lo expuesto aparece como muy posible la dimensión simbólica de los colgantes vinculada en varios casos con el mundo de los mágico o religioso, en tanto en cuanto puedan 
ser diferenciados estos campos, que en alguna ocasión puede ser entre otras cosas una exhibición de vinculaciones y poderes sobrenaturales que facilitan algunas cosas y que por otra parte protegen de varios males entre los cuales se encuentra el mal de ojo.

\section{¿Cuándo se puede hablar con seguridad de los objetos simbólicos?}

Excavaciones recientes en Marruecos y Sudáfrica y la revisión atenta de los materiales de antiguos trabajos arqueológicos en Argelia e Israel ha proporcionado un conjunto de datos de gran interés sobre la cuestión del simbolismo, ya que en varios de los yacimientos estudiados han aparecido varios objetos o estructuras a cada una de los cuales se le atribuye un valor simbólico ${ }^{3}$. El interés se ve aumentado por el hecho de que la reiteración de símbolos en un lugar avala este carácter de todos y cada uno de ellos y del conjunto.

Es frecuente que la redundancia se presente en lugares de especial significado y función, para que mediante esta técnica de expresión quede señalado del modo más claro posible el carácter especial del lugar.

Dentro de la esfera de lo religioso este hecho es muy frecuente como sabe todo aquel que ha visitado templos, cementerios, lugares sagrados o visto o participado en rituales de esta dimensión.

En los casos que aquí se estudian no se trata de santuarios, donde la misma idea se repita una y otra vez de diversos modos, sino de yacimientos donde elementos considerados simbólicos por sí mismos y que hasta su época aparecían desconectados entre si se encuentra por primera vez asociados en yacimientos muy distintos y distantes como los siguientes"

\section{Blombos Cave.}

Cueva situada en la costa del mar Índico en el sudeste de la República Sudafricana donde han aparecido, entre otros materiales novedosos como la industria lítica y ósea técnicamente elaborada, conchas del gasterópodo marino Nassarius kraussianus, fragmentos de ocre con huellas de uso, dos plaquetas de ocre con gra- bados geométricos. El yacimiento pertenece a la cultura arqueológica de Stilbay de la Middle Stone Age o Edad Media de la Piedra de África. Su cronología estimada por varios métodos es de alrededor de hace 75.000 años.

\section{Skhul.}

En la cueva de Skhul en Israel han aparecido cuentas perforadas de un gasterópodo marino el Nassarius gibbosulus impregnados de ocre, posiblemente algunos de ellos están relacionados con un área funeraria, pertenecientes al Paleolítico Medio del Levante o Próximo Oriente. Su cronología obtenida por diversos medios oscila entre hace 100.000 y 135.000 años.

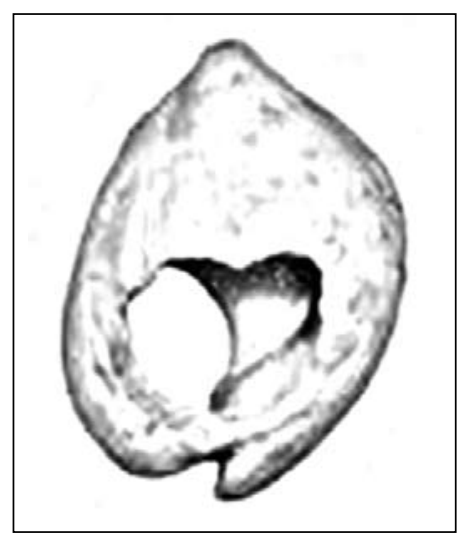

Concha del gasterópodo Nassarius gibbulosus perforada artificalmente. Procede de la cueva de Es Skhul, Israel (Wikimedia Commons)

\section{Oued Djebabna.}

En este lugar, con industria Ateriense, en el interior de Argelia han aparecido restos de cuentas de collar de Nassarius gibbosulus procedentes de una distancia mínima de 200 kilómetros, donde se encuentra la costa. Su cronología determinada por el carbono catorce es mayor de 35.000 años y de acuerdo con la comparación de yacimientos mejor datados con una industria semejante se le pueden atribuir con cierta seguridad unos 90.000 años.

\section{Qafzeh en Israel.}

En la cueva de este nombre, que contiene una industria del tipo del Paleolítico Medio del 
Próximo Oriente, con una cronología de unos 100.000 años, se han recogido cuentas del bivalvo marino Glycimeris insubrica, parte de ellas con perforaciones en el umbo. Estas conchas recogidas muertas y con una perforación natural que ha sido retocada en dos ejemplares proceden de la orilla del Mediterráneo que en la época en que se usaron estaba distante unos cuarenta kilómetros del yacimiento. Los ejemplares estaban impregnados de ocre, algunos en ambas caras, y en algún caso se le atribuido a algún ejemplar su función como recipiente de este pigmento, que en este yacimiento aparece en dos colores distintos rojo y amarillo.

\section{Taforalt en Marruecos.}

En la cueva des Pigeons, situada en el interior de Marruecos en un contexto con industria Ateriense con una antigüedad de unos 82.000 años, se han recogido un numeroso grupo de cuentas de collar obtenidas de la perforación de la concha del gasterópodo marino, Nassarius gibbosulus procedentes de la costa Mediterránea distante en la actualidad unos cuarenta kilómetros. Algunas de ellas están impregnadas de ocre.

\section{Valoración global}

Como puntos de mayor interés para una valoración conjunta de los hallazgos citados como elementos simbólicos hay que destacar que:

Aparecen conchas marinas perforadas usadas como colgantes en una franja cronológica cuyos extremos oscilan entre los 75.000 y los 130.000 años en yacimientos que en algún caso distan entre sí 5.000 kilómetros.

A pesar de la distancia entre ellos hay la pauta común de usar conchas de una única especie que en tres de los cinco yacimientos es la misma el Nassarius gibbosulus, en otro caso el del Nassarius kraussianus, se trata de un gasterópodo del mismo género de los Nassarius pero de distinta especie, que en todo caso es muy semejante en su forma y dimensiones a los ejemplares de los otros tres yacimientos. Se trata de gasterópodos de pequeño tamaño, alrededor de unos dos centímetros.

Aparecen manchadas o impregnadas de ocre, en varios de los lugares citados.
Son especies no comestibles entre otras cuestiones por su pequeño tamaño que hace muy difícil extraer la vianda de su interior y que han llegado a los yacimientos salvo en el caso de Blombos Cave, que está a la orilla del mar, desde distancias que van de los cuarenta a los doscientos kilómetros.

La mayoría tienen perforaciones, bien posiblemente naturales debidas a gasterópodos marinos de su mismo género, o artificiales cuidadosamente hechas, lo que indica su cuidadosa selección y manipulación en el caso de que el agujero para pasar el hilo de suspensión haya sido hecho artificialmente.

\section{¿Por qué se habla del carácter simbólico de las conchas?}

Hay numerosos datos del Paleolítico superior donde por diversos motivos entre los que se encuentra su asociación con otros elementos simbólicos, la larga distancia que han recorrido desde el lugar de recogida hasta el del hallazgo, su escaso o nulo valor alimenticio, el contexto donde se encontraron y los numeroso paralelos del valor simbólico de las conchas en culturas muy variadas, permiten pensar que es probable su carácter simbólico.

En el caso que nos ocupa hubo una rigurosa selección de las especies recogidas por cuanto estas comparten sus hábitats con otras muchas especies de gasterópodos y bivalvos, a mayores de muchos animales marinos.

La distancia al mar de los hallazgos pudo deberse a una recogida directa por parte de sus usuarios, cosa posible en los yacimientos a cuarenta kilómetros de la costa pues la expedición podría tardar día y medio o dos días de ida y otro tanto de vuelta o bien haber llegado a sus manos a través de una cadena de intercambio de bienes de prestigio, entre los que suelen encontrarse elementos que también tienen carácter simbólico a menudo asociados con la esfera de lo religioso.

Su asociación con el ocre, otro elemento que a mayores de su utilidad práctica variada, es un elemento de carácter simbólico refuerza la interpretación anterior por cuanto la redundancia de símbolos es una forma de destacar claramente su valor simbólico. 
Algunos investigadores sostienen, que el ocre que las colorea puede deberse no al hecho de que las conchas hayan sido teñidas intencionalmente sino de que se trata de una contaminación con este pigmento destinado a otros posibles diversos fines usados en el mismo yacimiento. También hay quienes mantienen la teoría de que el ocre en las conchas puede deberse a que alguna de ellas fue empleada como un simple recipiente sin mayores significaciones.

Sobre estas interpretaciones, que a mayores de su cautela pueden mostrar el viejo prejuicio eurocéntrico de que el carácter simbólico de estos elementos sólo aparece en el Paleolítico Superior de Europa se pueden hacer tres reflexiones de interés:

Si la mezcla del ocre con las conchas no es intencional, no por ello deja de tener valor el hecho de que dos elementos presuntamente simbólicos, vinculados a menudo con lo religioso, se usen al mismo tiempo en el mismo yacimiento, aunque sea con fines y aplicaciones diferentes sobre elementos distintos.

En este tipo relación se apoyarían mutuamente los dos elementos en su categoría simbólica que cada uno tenía ya por separado.

Si la asociación es intencional, como parece con claridad en algunos de estos y otros yacimientos de la época, tendríamos reforzada la teoría de la redundancia de lo simbólico, de que la unión intencionada de varios elementos simbólicos refuerza el carácter del todo y de todos y cada uno de los elementos con este posible carácter.

Una tercera posibilidad es la de que un elemento pueda tener varias funciones alternativas o complementarias.

Así, frente a los que argumentan el valor funcional de ocre como pegamento o masilla de algunos objetos en la Edad Media de la Piedra de África, se puede argumentar que también es posible que aparte de su adherencia le haya aportado un valor simbólico al artefacto en que se ha usado.

En este caso podrían haber servido también para favorecer la adherencia de las conchas o bien para teñirlas de un color distinto al natural.
La posible vinculación con el mundo funerario sería un indicativo de su relación con otro ámbito al que tradicionalmente se le atribuye una dimensión simbólica. De ser esto así se repetiría un caso de redundancia como hemos visto en la asociación explicitada anteriormente de cuentas más ocre.

Otra cuestión, y muy importante, es que las conchas han sido utilizadas como colgantes, con lo cual han tenido que estar relacionadas con varias de las implicaciones simbólicas que tienen en las diferentes culturas del pasado y del presente este tipo de objetos.

De acuerdo con lo expuesto podemos concluir, al menos si no como teoría sí como hipótesis de trabajo plausible, que las conchas usadas como cuentas de collar tenían funciones simbólicas posiblemente relacionadas con lo social y lo religioso.

La procedencia de las conchas que en lugar donde han sido halladas son un objeto exótico muy posiblemente tenga una significación especial relacionada y dada por este carácter como ocurre en materiales exóticos documentados por la historia y la etnología.

Proceden de lo otro, de un lugar distante y distinto y son unos animales desconocidos por su forma, color, hábitos, hábitats, en el territorio que normalmente utilizan sus usuarios.

El espacio distante y distinto, desconocido o muy poco conocido y poco accesible independientemente del motivo, suele estar dotado de propiedades reales o imaginarias distintas al hogar y ocupado por seres que tienen propiedades diferentes a las del mundo habitual y cotidiano.

En algunos casos ambos tienen caracteres positivos, por ejemplo, son el país de las maravillas donde ocurren portentos positivos y hay seres excepcionales o bien es el mundo de los muertos o de ciertos entes de tipo religioso.

El caso contrario es el del espacio exótico y de los objetos procedentes de él que son considerados como un lugar negativo como por ejemplo el infierno, el límite del mundo, lleno de todo tipo de peligros, de monstruos perversos, demonios, ogros, animales fantásticos, que a menudo también hacen una referencia a poderes malignos vinculados con la esfera de lo religioso. 
En estos lugares hay plantas, animales y minerales que tiene propiedades singulares relacionadas con la esfera de lo simbólico.

Por tanto los materiales que proceden de mundo distinto tiene propiedades relacionadas con su origen algunas de las cuales pueden ser desconocidas en donde los usaron. De hecho las conchas por su simetría y color despiertan una emoción estética debido a mecanismos neurológicos que comienzan a entenderse relativamente bien desde que se aplican los conocimientos de las neurociencias a la interpretación del comportamiento humano actual y en el pasado.

El prestigio del poseedor de este bien escaso y preciado debía de ser grande no solo por su dimensión estética sino por los valores asociados con su lugar de origen.

Si hubiesen sido recogidos directamente por ellos habría que añadir que a mayores sería también la señal o aviso de que su portador ha realizado una aventura: un viaje peligroso a un mundo diferente al habitual. Este hecho es una afirmación de la capacidad de su portador lo que podría darle una posición social relativamente importante: la del poseedor de un bien valioso por su escasez, dimensión estética y poderes, o la de una persona con capacidad para afrontar exitosamente aventuras que encierran muchos peligros reales e imaginarios.

\section{NOTAS}

1 F. D’Errico, "Additional evidence on the use of personal ornaments in the Middle Paleolithic of North Africa", PNAS Sep 22, 2009; 106(38):1605116056.

D. E. Bar-Yosef, B. Vandermeerch and O. Bar-Yosef, "Shells and ochre in Middle Paleolithic Qafzeh Cave: indications for modern behavior". Journal of
Human Evolution 56(2009) 307-314.

L.S. Dubin, The history of beads: From 100.000 B.C. to the Present. Revised and Expanded Edition. Abrams. New York. 2009

M. Vanharen, "Thinking strings: additional evidence for personal ornament use in the Middle Stone Age of Blombos Cave, South Africa", Journal of Human Evolution, 64,6: 500-517. June 2013.
${ }^{2}$ N.A. Léo Neto, R. A Voeks, T.LP Días and R. RN Alves," Mollusks of Candomblé: symbolic and ritualistic importance", Journal of Ethnobiology and Ethnomedicine 2012, 8:10. May 2012.

${ }^{3}$ Vide nota 1 